OPEN ACCESS

Edited by:

Choong-Min Ryu,

Korea Research Institute

of Bioscience and Biotechnology,

South Korea

Reviewed by:

Raquel Campos-Herrera, University of the Algarve, Portugal Zonghua Wang,

Fujian Agriculture and Forestry University, China

${ }^{*}$ Correspondence: Yongjun Zhang

yjzhang@ippcaas.cn: yjippc@126.com

Specialty section: This article was submitted to Plant Microbe Interactions, a section of the journal

Frontiers in Plant Science

Received: 24 October 2016 Accepted: 19 January 2017

Published: 03 February 2017

Citation

Sun $Y$, Huang $X Z$, Ning $Y S$, Jing $W X$, Bruce TJA, Qi FJ, Xu QX, Wu KM, Zhang YJ and Guo YY (2017) TPS46, a Rice Terpene Synthase Conferring Natural Resistance to Bird Cherry-Oat Aphid, Rhopalosiphum padi

(Linnaeus). Front. Plant Sci. 8:110. doi: $10.3389 / f p / s .2017 .00110$

\section{TPS46, a Rice Terpene Synthase Conferring Natural Resistance to Bird Cherry-Oat Aphid, Rhopalosiphum padi (Linnaeus)}

\author{
Yang Sun 1,2, Xinzheng Huang ${ }^{1}$, Yuese Ning', Weixia Jing ${ }^{1,3}$, Toby J. A. Bruce ${ }^{4}$, \\ Fangjun Qi ${ }^{1}$, Qixia X ${ }^{1}$, Kongming $W^{1}{ }^{1}$, Yongjun Zhang ${ }^{1 *}$ and Yuyuan Guo ${ }^{1}$
}

\begin{abstract}
${ }^{1}$ State Key Laboratory for Biology of Plant Diseases and Insect Pests, Institute of Plant Protection, Chinese Academy of Agricultural Sciences, Beijing, China, ${ }^{2}$ Institute of Plant Protection, Jiangsu Academy of Agricultural Sciences, Nanjing, China, ${ }^{3}$ College of Plant Protection, Shandong Agricultural University, Tai'an, China, ${ }^{4}$ Department of Biological Chemistry and Crop Protection, Rothamsted Research, Harpenden, UK
\end{abstract}

Plant terpene synthases (TPSs) are key enzymes responsible for terpene biosynthesis, and can play important roles in defense against herbivore attack. In rice, the protein sequence of TPS46 was most closely related to maize TPS10. However, unlike maize tps 10, tps46 was also constitutively expressed in rice even in the absence of herbivore attack. Potential roles or constitutive emissions of specific volatiles may due to the constitutive expressions of tps 46 in rice. Therefore, in the present study, RNA interference $(\mathrm{Ri})$ and overexpression $(\mathrm{Oe})$ rice lines were generated to investigate the potential function of TPS46 in Oryza sativa sp. japonica. Interestingly, the rice plants become more susceptible to Rhopalosiphum padi when expression of tps 46 was silenced compared with Wt in greenhouse conditions. Artificial infestation bioassays further confirmed that Ri rice lines were susceptible to $R$. padi, whereas Oe rice lines were repellent to $R$. padi. Based on GC-MS and ToF-MS analysis, a total of eight volatile products catalyzed by TPS46 in rice were identified. Among them, only limonene and $\mathrm{E} \beta \mathrm{f}$ could be detected in all the Ri, Oe, and Wt lines, whereas other six volatiles were only found in the blend of volatiles from Oe lines. Moreover, the amount of constitutive limonene and $\mathrm{E} \beta \mathrm{f}$ in the $\mathrm{Ri}$ lines was significantly lower than in Wt lines, while the amounts of these two volatiles in the Oe line were obviously higher than in control rice. Our data suggested that the constitutive emissions of $\mathrm{E} \beta \mathrm{f}$ and limonene regulated by the constitutive expression of tps 46 may play a crucial role in rice defense against $R$. padi. Consequently, tps 46 could be a potential target gene to be employed for improving the resistance of plants to aphids.

Keywords: insect-plant interactions, Rhopalosiphum padi, tps46, (E)- $\beta$-farnesene, terpene syntheses

\section{INTRODUCTION}

Plant terpenoids have ecological functions in mediating plant interactions and in allowing them to withstand biotic and abiotic stress (Pichersky and Gershenzon, 2002; Degenhardt et al., 2003). Volatile terpenes can help plants attract pollinators and predators of herbivores (Pichersky and Gershenzon, 2002; Degenhardt et al., 2003; Lou et al., 2005, 2006; Yuan et al., 2008). Additionally, 
terpenoid phytoalexins may be involved in the defense against herbivores (Balkema-Boomstra et al., 2003). When attacked by a herbivore, many plants are able to initiate indirect defenses by synthesizing and releasing complex blends of volatile terpenes that attract natural enemies of the herbivore (Turlings et al., 1991; Turlings and Ton, 2006; Yuan et al., 2008; Degenhardt, 2009). In grasses, especially in rice and maize, the biosynthesis of volatile terpenes regulated by terpene synthase ( $t p s$ ) genes has been investigated in details (Degenhardt, 2009), and many grass TPSs play crucial roles in the biosynthesis of plant herbivore-induced volatiles, such as TPS1, TPS10, and TPS23 in maize (Schnee et al., 2002, 2006; Köllner et al., 2008), as well as Os02g02930, Os08g07100 (TPS46), TPS3, and Os08g04500 in rice (Cheng et al., 2007; Yuan et al., 2008).

In our previous work, we found that a candidate gene, SSBis039 (Os08g0167800), was significantly upregulated in rice after Chilo suppressalis (Lepidoptera: Pyralidae) infestation for $24 \mathrm{~h}$ (Sun et al., 2011). Because there was 100\% identity between SSBis039 and tps46, we confirmed that SSBis039 was the rice tps46. Rice tps46 is known to be an important gene in defense against Spodoptera frugiperda (Lepidoptera: Noctuidae) and C. suppressalis (Yuan et al., 2008; Sun et al., 2011). Phylogenetic analysis has shown that rice TPS46 is very closely related to maize TPS10 (Yuan et al., 2008). To investigate the roles of TPSs, a full-length cDNA of tps was usually cloned from herbivore-damaged plants, and then expressed in Escherichia coli or overexpressed in Arabidopsis thaliana (Beale et al., 2006; Schnee et al., 2006; Nagegowda et al., 2008; Yuan et al., 2008). By using farnesyl diphosphate (FPP) as the substrate, E. coli-expressed recombinant maize TPS10 catalyzed the formation of 11 sesquiterpenes, including $\alpha$-copaene, (E)- $\beta$-caryophyllene, $(E)$ - $\alpha$-bergamotene, sesquisabinene, $(E)$ $\beta$-farnesene (E $\beta f)$, germacrene, zingiberene, $\alpha$-muurolene, $\beta$-bisabolene; $\delta$-cadinene, and sesquiphellandrene (Schnee et al., 2006). However, only six volatile compounds, such as (E)- $\alpha$-bergamotene, sesquisabinene, Eßf, zingiberene, $\beta$-bisabolene, and sesquiphellandrene were significantly increased in tps10-overexpresing transgenic A. thaliana (Schnee et al., 2006).

Escherichia coli-expressed recombinant rice TPS46 converted FPP into a blend of 14 sesquiterpenes: 7-epi-sesquithujene, sesquithujene, $(Z)$ - $\alpha$-bergamotene, $(E)$ - $\alpha$-bergamotene, sesquisabinene $A, E \beta f$, sesquisabinene, $\gamma$-curcumene, an unknown sesquiterpene, zingiberene, $\beta$-bisabolene, $\beta$-curcumene, $\beta$-sesquiphellandrene, and (E)- $\gamma$-bisabolene (Yuan et al., 2008). Among these volatiles, $(E)$ - $\alpha$-bergamotene, sesquisabinene, E $\beta f$, zingiberene, $\beta$-bisabolene, and sesquiphellandrene were the same volatile compounds synthesized by both TPS10 and TPS46 in vitro (Schnee et al., 2006; Yuan et al., 2008). Interestingly, these six specific volatile compounds were also the main products in the tps 10 overexpressed A. thaliana (Schnee et al., 2006). However, the products regulated by TPS46 in rice plants were still unclear. Therefore, given the differences in products in different expression systems, intensive study (overexpression of tps46 in rice) was required in the present study to determine whether the products of TPS46 in vitro are consistent with that in vivo.
Previous studies revealed that tps10 was not expressed in maize in the absence of herbivore attack (Schnee et al., 2006), whereas the expression of tps 46 could be detected in rice even if the plants were not stimulated by any external factors (Yuan et al., 2008; Sun et al., 2011). Therefore, tps46 may play a different biological role in rice under the natural conditions. In recent years, RNA interference (RNAi) technique is widely used in transgenic plants to explore the function of target genes. In the present study, RNAi and overexpression trials were conducted to silence or overexpress tps46 in rice (Oryza sativa ssp. japonica "Nipponbare" NPB), and then investigate the biological roles of TPS46 under natural conditions or overexpression conditions. Consequently, our results provide an important foundation for understanding the function of TPS46 in rice sesquiterpenoid biosynthesis and plant defense.

\section{MATERIALS AND METHODS}

\section{Isolation of cDNA of Rice tps46}

The full-length cDNA of rice tps46 [previously named as SSBis039 and shown to be induced by $C$. suppressalis larvae (Sun et al., 2011)] was cloned from "NPB" rice leaf tissues using the SMARTer ${ }^{\text {TM }}$ RACE cDNA Amplification Kit (Clontech, Palo Alto, CA, USA) according to the protocol described in Sun et al. (2014). Two $5^{\prime} \mathrm{GSPs}\left(5^{\prime} \mathrm{GSP} 1\right.$ and $5^{\prime} \mathrm{GSP} 2$ ) and two $3^{\prime} \mathrm{GSP}$, ( $3^{\prime}$ GSP1 and $3^{\prime}$ GSP2) were designed using Primer 5.0 software based on the partial tps46 sequence obtained by our previous study (Sun et al., 2011), and primers were listed in Table $\mathbf{1}$.

\section{The tps46-RNAi and tps46-Overexpression Transgenic Trials}

Based on the sequence of tps46 (Genbank accession number: EU596452), the rice tps46 fragment was amplified using the

TABLE 1 | Primers used in the study.

\begin{tabular}{|c|c|}
\hline Primer name & Sequence $\left(5^{\prime}-3^{\prime}\right)$ \\
\hline \multicolumn{2}{|c|}{ tps46 fragment clone } \\
\hline RiTPS46-forward & TCAggtaccactagtCTACACGAAATGAAGCCATA \\
\hline RiTPS46-reverse & CATggatccgagctcAGTTCCAGGTGTTCCTCTAT \\
\hline \multicolumn{2}{|l|}{ tps46 gene clone } \\
\hline OeTPS46-forward & CATGTCATCGACACCTGCA \\
\hline OeTPS46-reverse & TTAAATGCTATATGGCTCAACG \\
\hline \multicolumn{2}{|l|}{$q R T-P C R$} \\
\hline TPS46-forward & TGAAGAGGCACTAGGTCCAAAC \\
\hline TPS46-reverse & CCATCCCAACTAAAGAAGCACA \\
\hline EF1 $\alpha$-forward & AGACGCACATCAACATCG \\
\hline EF1 $\alpha$-reverse & GAACTTCCACAGGGCAATA \\
\hline \multicolumn{2}{|l|}{ Race-PCR } \\
\hline TPS46-3'GSP1 & CACACGATGGTGGAAAGAGCTTAACGTTG \\
\hline TPS46-3'GSP2 & GACAGGAGCATGCTCGGAGCCCCATTA \\
\hline TPS46-5'GSP1 & CAAGCATCATGCTCTCCTCGGTTGTAGC \\
\hline TPS46-5'GSP2 & TGGACCTAGTGCCTCTTCAAATGAATCAA \\
\hline
\end{tabular}

The restriction sites used for clone are underlined and shown in bold. 
RiTPS46F and RiTPS46R primers (Table 1). The 535 bps PCR product was digested with both SacI and SpeI or with KpnI and $B a m H I$, gel purified, and then ligated into the same restriction sites within the RNAi ptck303 binary vector of rice (Wang et al., 2004). The ptck303-tps46 RNAi binary vector contained an antisense tps46 fragment, a rice intron, and sense tps46 fragment between the maize (Zea mays) ubiquitin promoter and the nos $3^{\prime}$ terminator in the ptck303 binary vector (Wang et al., 2004; Kong et al., 2006). For tps46 overexpression, the full-length CDS of tps46 was amplified using the OeTPS46F and OeTPS46R primers (Table 1). Then, the $1641 \mathrm{bp}$ of tps46 was ligated to a pCXUN vector with the TA cloning system (Chen et al., 2009). Rice callus was induced from the embryos of mature seeds of the Japonica cultivars "NPB" using an Agrobacterium tumefaciens-mediated method (Qu et al., 2006; Park et al., 2012). Finally, more than 15 T0 tps46-RNAi ( $\mathrm{Ri})$ rice lines and 15 T0 tps46-Overexpression (Oe) lines were obtained. The segregation lines of $\mathrm{Ri}$ and $\mathrm{Oe}$ transgenic plants were set as RWt or OWt controls.

\section{Insect Population Survey on T1 Ri Lines}

Seven T1 Ri line (Ri1, 3, 4, 5, 8, 10, 11) and three T1 RWt line (RWt1, 2, 3) rice seedlings were first grown in a MS plus (75 mg L-1, Hygromycin B) selective liquid medium for 3 days in an incubator $\left(28^{\circ} \mathrm{C} / 26^{\circ} \mathrm{C}, 16 \mathrm{~h}\right.$ of light $/ 8 \mathrm{~h}$ of dark, and $50 \%$ humidity, respectively). Rice seedlings were grown individually in a cylindrical pot $(25 \mathrm{~cm}$ and a height of $30 \mathrm{~cm})$ in a greenhouse with temperature ranging from 24 to $32^{\circ} \mathrm{C}, 16 \mathrm{~h}$ of light $/ 8 \mathrm{~h}$ of dark, and $50 \%$ humidity, where aphids, such as Rhopalosiphum padi (Hemiptera: Aphididae), Myzus persicae (Hemiptera: Aphididae), Brevicoryne brassicae (Hemiptera: Aphididae), and Aphis gossypii (Hemiptera: Aphididae), occurred naturally. The quantitative real-time PCR (qRT-PCR) measurements were conducted to investigate the target mRNA transcripts in leaves and sheaths of rice seedlings at the tillering stage. After qRTPCR, every rice line as one treatment was random placed in a greenhouse to evaluate the insect infestation when exposed to aphids. All treatments in the experiment were repeated three times. This survey experiment was repeated twice. Insect infestation was recorded every day between 15:00 and 17:00 from tillering to the grain-filling stage of the rice plants. Aphid species were identified in insect taxonomy and toxicology laboratory, China Agricultural University.

\section{Bioassay of R. padi Performance on Plants}

Newly emerged wingless $R$. padi colonies were kindly provided by Professor Xi-wu Gao of the insect toxicology laboratory, China Agricultural University, which had been maintained in the absence of insecticide exposure more than 10 years ( $\mathrm{Lu}$ et al., 2013). The different rice lines were cultivated as described in the previous section "insect population survey on T1 Ri lines." Before bioassay, expression of tps46 in the Ri3, Ri5, Ri8, Ri10, RWt, Oe6, Oe7, Oe9, Oe11, and OWt rice lines at jointingbooting stage was evaluated with qRT-PCR measurements. Soon after, Ri3, Ri5, Ri8, Ri10, Oe6, Oe7, Oe9, and Oe11 were selected for indoor bioassays in an insect-free greenhouse (Figure 2 and
Supplemental Figure S1). The temperature in the greenhouse was kept at $22-28^{\circ} \mathrm{C}$ and artificial infestation was performed between 13:00 and 17:00 PM. Each rice seedling in each pot was infested with 40 newly emerged wingless $R$. padi, and the number of $R$. padi on each rice seedling was recorded after 24,48 , and $72 \mathrm{~h}$. Each treatment was replicated 3-5 times.

\section{qRT-PCR Analysis of tps46 in Different Rice Strains}

Total RNA from the leaves and sheaths of rice seedlings (Oe, $\mathrm{Ri}$, and $\mathrm{Wt}$ ) was extracted by using the SV Total RNA Isolation System (Promega, Madison, WI, USA). One microgram of total RNA was used as a template for synthesizing the first-strand cDNA following the manufacturer's protocol for MMLV Reverse Transcriptase (Promega, Madison, WI, USA). The cDNAs were treated with Ribonuclease H (TaKaRa, Tokyo, Japan) and quantified on a ND-1000 spectrophotometer (NanoDrop, Wilmington, DE, USA) at $\mathrm{OD}_{260} \mathrm{~nm}$. The qRT-PCR was performed using the SYBR Premix Ex Taq Kit (TaKaRa, Tokyo, Japan) on a Bio-rad iCycler detecting system with SYBR green fluorescent dye. The rice EF-1 $\alpha$ gene (Genbank accession number: AK061464) was used as an internal control to normalize the transcript levels of tps46 in each experiment (Jain et al., 2006), and the specific primers of the target gene and reference gene are listed in Table 1. The qRT-PCR reactions were replicated five times each treatment and non-template control (NTC) reactions were performed in triplicate. The $2^{-\Delta \Delta \mathrm{Ct}}$ method was used to evaluate the relative expression of the target gene (Livak and Schmittgen, 2001).

\section{Volatile Collection}

Rice lines, including Oe6, Oe7, OWt, Ri5, Ri8, and RWt at the beginning of jointing-booting stage were selected for volatile collection by an open head-space sampling system (Yu et al., 2010). Pots containing one rice plant were placed within a cylindrical type glass container $(25 \mathrm{~cm}$ in diameter $\times 50 \mathrm{~cm}$ high). The container was sealed with a glass lid that had an air inlet and an air outlet. The container was tightly sealed with metal clamps on the lid. Air, purified by passage through an activated charcoal filter, was actively pumped through the container at a flow rate of $1 \mathrm{ml} / \mathrm{min}$ with a vacuum pump (Beijing Institute of Labor Instrument, Beijing, China). Volatiles were collected for $6 \mathrm{~h}$ on $60 \mathrm{mg}$ of 60/80 mesh Tenax-TA (Shanghai ANPEL Scientific Instrument Company, Shanghai, China) in an 8-mm-diameter glass tube, which was directly connected to the outlet. All connections were made with Teflon tape. Three to five replicates were performed for each experiment (Huang et al., 2015). All collections were conducted from 11:00 AM to 17:00 $\mathrm{PM}$. The collected samples were stored at $4^{\circ} \mathrm{C}$ for further analysis.

\section{Gas Chromatography-Mass Spectrometry (GC-MS) Analyses}

Volatiles from Ri5, Ri8, and RWt rice lines were extracted with $300 \mu \mathrm{l}$ of HPLC-grade hexane (Fisher Scientific, Fairlawn, NJ, USA), and 21 ng n-octane (Sigma-Aldrich, Oakville, Canada) was individually added as an internal standard. The collected samples 
were analyzed by Agilent 6890 GC coupled with an Agilent 5973 MS detector on a HP-5MS dimethylpolysiloxane column $(30 \mathrm{~m} \times 0.250 \mathrm{~mm} \times 0.25 \mu \mathrm{m}$, Agilent Technologies, Palo Alto, CA, USA). One microliter of the sample was used for injection by using a $10 \mu \mathrm{l}$ syringe (Hamilton, USA). The GC oven temperature was maintained at $40^{\circ} \mathrm{C}$ for $2 \mathrm{~min}$ and increased to $100^{\circ} \mathrm{C}$ at a rate of $6^{\circ} \mathrm{C} / \mathrm{min}$ (hold for $1 \mathrm{~min}$ ), increased again at a rate of $5^{\circ} \mathrm{C} / \mathrm{min}$ to $150^{\circ} \mathrm{C}$ (hold for $1 \mathrm{~min}$ ), increased to $250^{\circ} \mathrm{C}$ at a rate of $10^{\circ} \mathrm{C} / \mathrm{min}$, and finally maintained at $250^{\circ} \mathrm{C}$ for $5 \mathrm{~min}$. Helium was used as the carrier gas at $1.0 \mathrm{ml} / \mathrm{min}$. Tentative identifications were made by comparison of mass spectra (a) with mass spectra libraries (NIST and Department of Chemical Ecology, Göteborg University, Sweden) and (b) with the mass spectra and retention times of authentic samples obtained from Fluka, Sigma ${ }^{1}$.

Volatiles from the Oe6, Oe7, and OWt rice plants adsorbed on Tenax-TA were eluted with $300 \mu$ l HPLC-grade hexane (Fisher, Fairlawn, NJ, USA), and 2.586 ng of ethyl caprate (Sigma-Aldrich, Oakville, Canada) was individually added as an internal standard. Comprehensive two-dimensional gas chromatography/time-offlight mass spectrometry (GC x GC-ToF, Pegasus 4D, LECO, USA) was performed to analyze the volatile samples. The GC inlet and transfer line were held constant at $250^{\circ} \mathrm{C}$. A $1 \mu \mathrm{l}$ injection was made onto column 1 (Rxi-5Sil MS, $30 \mathrm{~m} \times 0.25 \mathrm{~mm}$ i.d. $\times 0.25 \mu \mathrm{m}$, Restek). The effluent from column 1 was then transferred to column 2 (Rtx-200, $2 \mathrm{~m} \times 0.180 \mathrm{~mm}$ i.d. $\times 0.2 \mu \mathrm{m}$, Restek). Column 1 was held at $50^{\circ} \mathrm{C}$ for $1 \mathrm{~min}$ followed by a two-step temperature increase, first to $150^{\circ} \mathrm{C}$ (at a rate of $5^{\circ} \mathrm{C} / \mathrm{min}$, hold for $2 \mathrm{~min}$ ) and then to $250^{\circ} \mathrm{C}$ (at a rate of $10^{\circ} \mathrm{C} / \mathrm{min}$, hold for $2 \mathrm{~min}$ ). Column 2 was held at $55^{\circ} \mathrm{C}$ for $1 \mathrm{~min}$ followed by a two-step temperature increase to $155^{\circ} \mathrm{C}$ (at a rate of $5^{\circ} \mathrm{C} / \mathrm{min}$ with a $2 \mathrm{~min}$ hold) and then to $255^{\circ} \mathrm{C}$ (at a rate of $10^{\circ} \mathrm{C} / \mathrm{min}$, with a $2 \mathrm{~min}$ hold). The volatile products were identified by comparing their retention times and mass spectra areas to authentic standards (Gaquerel et al., 2009; Huang et al., 2015).

\section{Data Analysis}

Differences of tps46 expression levels, R. padi numbers and volatile emission between different rice lines were analyzed using one-way ANOVA methods by SAS 9.0 software for Windows with Duncan's new multiple range method $(P<0.05)$ (SAS 9.0 system for windows, 2002, SAS Institute Inc., Cary, NC, USA).

\section{RESULTS}

\section{R. padi Was the Dominant Aphid Species on the tps46-RNAi Rice Plants}

In our greenhouse, several aphid species on rice lines were observed and recorded, while only the significant difference in $R$. padi numbers between the $\mathrm{Ri}$ and $\mathrm{RWt}$ rice plants were observed. Other aphids tested included $M$. persicae, B. brassicae, and A. gossypii. In infestation trials, a small number of winged $R$. padi were found on rice plants in the late tillering stage. Then the wingless $R$. padi population sharply increased on

${ }^{1}$ http://www.sigmaaldrich.com/

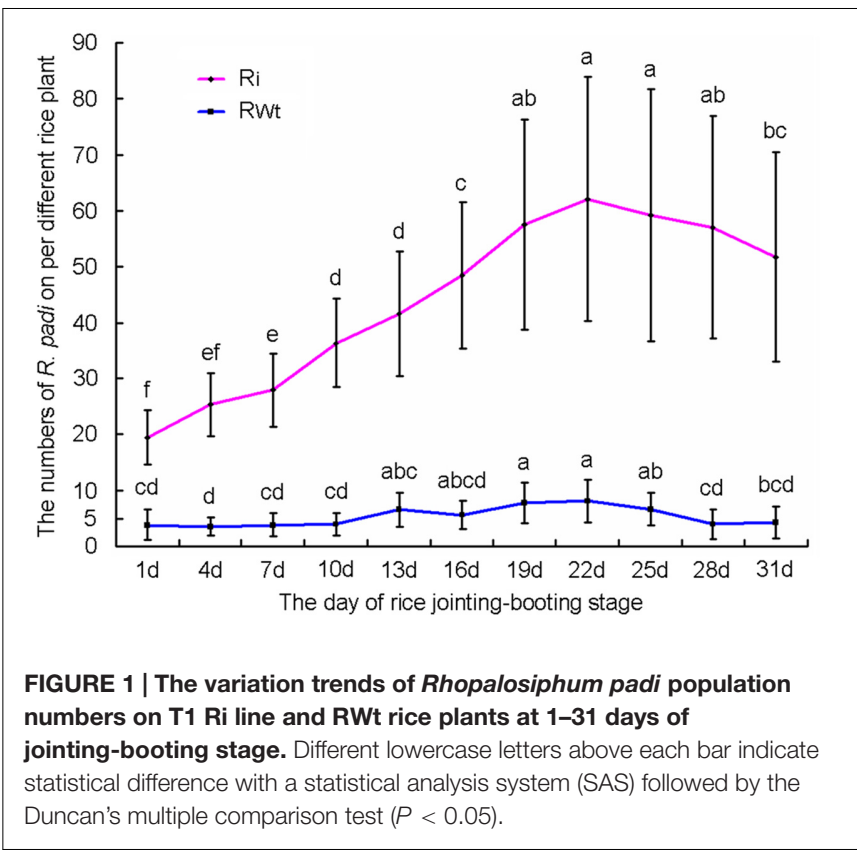

$\mathrm{Ri}$ rice lines in the jointing-booting stage and the population reached a peak value (62 \pm 22 aphid/ individual Ri plant) at 22 days into the jointing-booting stage (Figure 1). However, the $R$. padi population on the segregation lines of Ri transgenic plants (RWt) increased very little during the same stage and after 31 days was not significantly different the population size at the start (Figure 1). Also, the same tendency in this experiment was reproduced in another repeats (Figure $\mathbf{1}$ and Supplemental Figure S3). Moreover, $R$. padi populations on all seven tested $\mathrm{Ri}$ rice lines were significantly higher than on control rice lines $(P<0.05)$. In contrast, there was no remarkable difference in $R$. padi populations among the three RWt control rice lines $(P>0.05)$ (Figure 2A). Expression levels of tps46 were significantly lower in all the seven tested Ri rice lines than in control lines $(P<0.05)$, and there was no visible difference between the RWt control rice lines $(P>0.05)$ (Figure 2B). The $R$. padi population ( $83 \pm 22$ aphid/ individual Ri plant) on Ri5 rice plants among these $\mathrm{Ri}$ rice lines was significantly higher than on Ri1, Ri4 and Ri11 rice lines $(P<0.05)$ (Figure $2 \mathrm{~A}$ ). Interestingly, the expression of tps46 in the Ri5 line was only $6.81 \pm 0.79 \%$ of the expression in control rice lines (Figure 2B). In addition, our observations have shown that there was no $R$. padi on control rice lines in the field or the greenhouse for many years until the Ri rice plants were cultivated.

\section{Artificial Infestation Bio-Assays of Different Rice Lines}

The tps46 expression levels in different rice lines were analyzed by qRT-PCR before the $R$. padi infestation bio-assays. The expression of tps46 in all of the Oe rice lines was significantly higher than in the $\mathrm{Ri}$ and control rice lines $(P<0.05)$, whereas expression of tps46 in all of the Ri rice lines was obviously lower than expression in the control rice lines $(P<0.05)$. Additionally, 


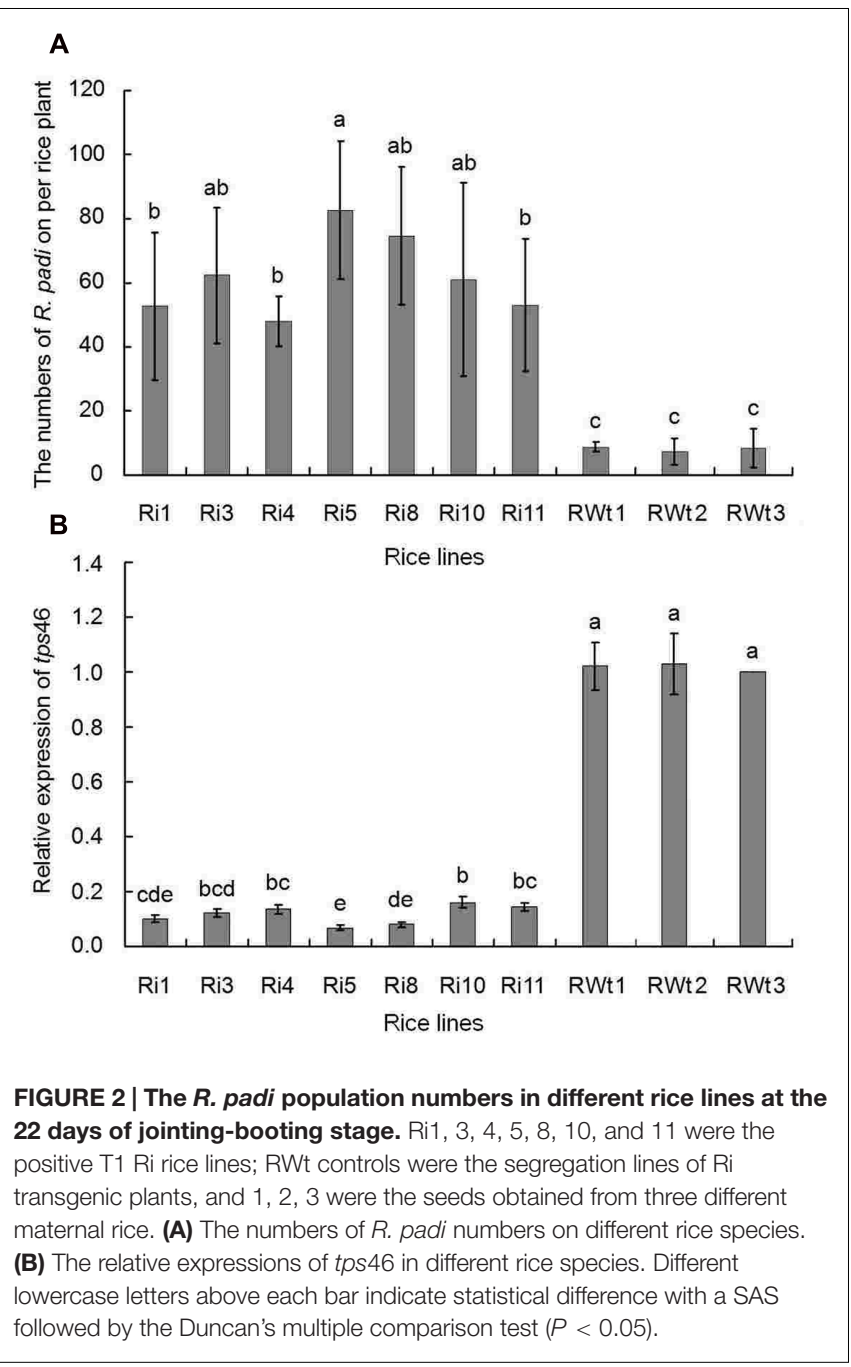

there was no significant difference between the RWt and OWt control rice lines $(P>0.05)$ (Figure 3D).

The survival rate of $R$. padi on Ri rice lines was significantly higher than that on Oe and control lines $(P<0.05)$. The survival rate of $R$. padi on the Oe lines was significantly lower than the survival rate on control rice plants $(P<0.05)$, and there was no obvious difference in the $R$. padi survival rate between RWt and OWt control rice lines $(P>0.05)$ (Figures 3A-C). The survival rate of $R$. padi on the two control lines was only approximately $20 \%$ after $72 \mathrm{~h}$. The survival rate of $R$. padi on the Ri lines was higher than 50\% during the same period and reached $67 \pm 11.5 \%$ on Ri5 rice plants (Figure $3 \mathrm{C}$ ). During the same period, the survival rate of $R$. padi on the Oe rice lines was below $10 \%$ t after $72 \mathrm{~h}$, and was less than $5 \%$ on the Oe6 and Oe7 rice plants (Figure 3C). Interestingly, the expression of tps46 in the Oe6 and Oe7 rice plants was $837 \pm 144$ and $976 \pm 178$ times higher than in control lines. The expression was significantly higher compared to expression in the other tested rice plants $(P<0.05)$. However, the expression of $t p s 46$ in the Ri5 lines was only $5.1 \pm 0.94 \%$ of that in control rice lines (Figure 3D).

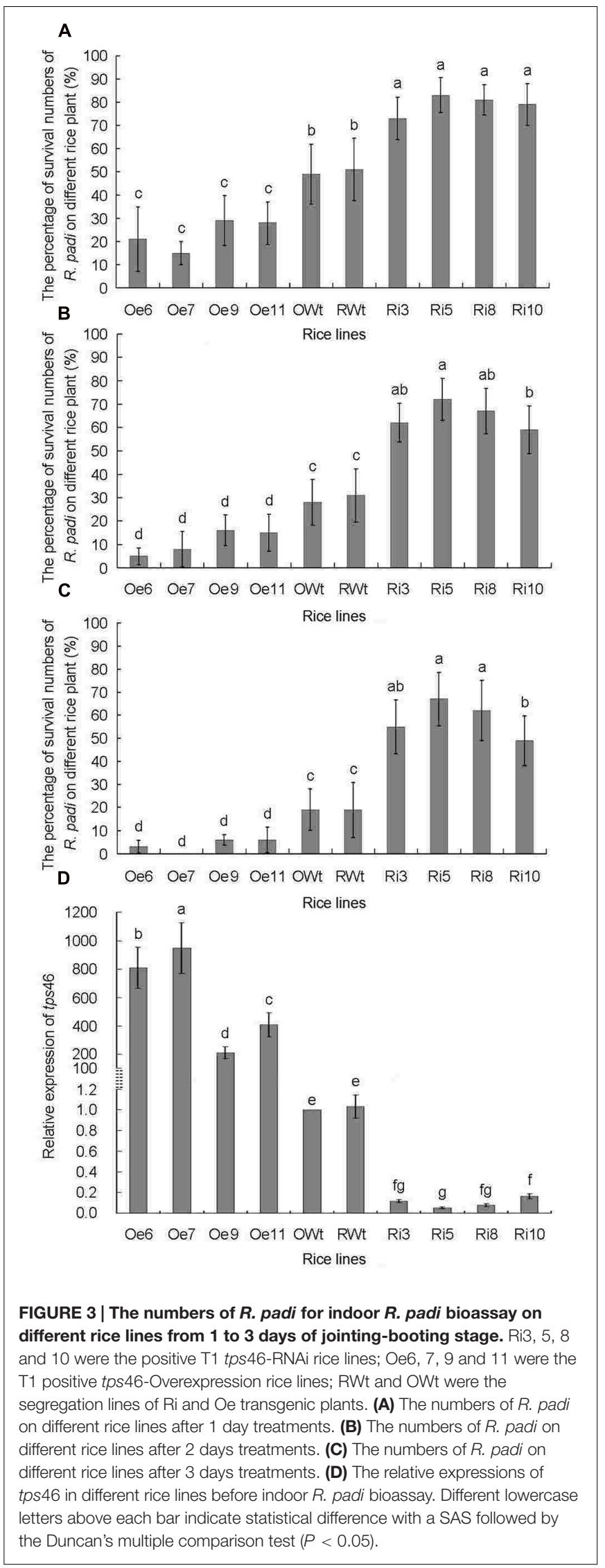




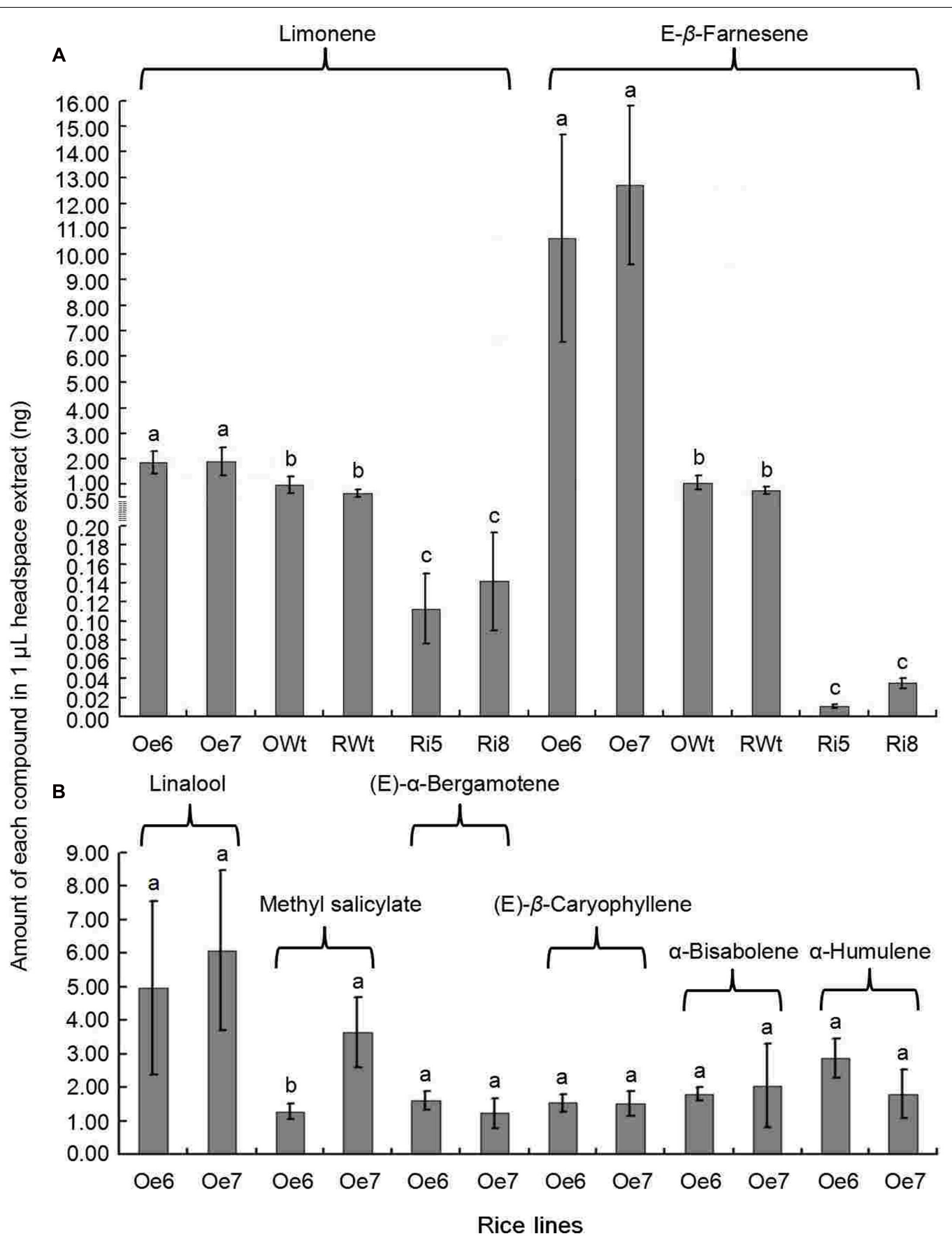

FIGURE 4 | A mount of each compound in $\mathbf{1} \boldsymbol{\mu} \mathbf{L}$ headspace extract of different rice lines. Ri5 and 8 were the positive $T 1$ Ri rice lines; Oe6, and 7 were the positive T1 Oe rice lines; RWt and OWt were the segregation lines of Ri and Oe transgenic plants. (A) Amount of limonene and (E)- $\beta$-farnesene in different rice lines. (B) Amount of linalool, methyl salicylate, $(E)$ - $\alpha$-bergamotene, $(E)$ - $\beta$-caryophyllene, $\alpha$-bisabolene, and $\alpha$-humulene in different positive Oe transgenic rice lines. Different lowercase letters above each bar indicate statistical difference with a SAS followed by the Duncan's multiple comparison test $(P<0.05)$.

\section{Volatile Emission from Ri and Oe Rice Plants}

Recombinant TPS46 protein can catalyze substrates to produce several terpene volatiles in vitro (Yuan et al., 2008). In this study, we further investigated the volatile compounds released from the
$\mathrm{Ri}$, Oe, and Wt rice lines. GC-MS analyses indicated that the amount of two volatile compounds, limonene and E $\beta$ f, decreased significantly in the Ri rice lines compared to the RWt (control) rice plants $(P<0.05)$ (Supplemental Figure S2 and Figure 4A). The amounts of limonene in $1 \mu \mathrm{L}$ of extracts from Ri5, Ri8, and RWt rice headspace samples were $0.11 \pm 0.04,0.14 \pm 0.05$, and 
$0.65 \pm 0.14 \mathrm{ng}$, respectively. The amounts of E $\beta \mathrm{f}$ in $\mathrm{Ri5}, \mathrm{Ri} 8$, and RWt rice headspace samples were $0.01 \pm 0.002,0.035 \pm 0.005$, and $0.77 \pm 0.13 \mathrm{ng}$, separately (Figure 4A). As expected, the amount of limonene and $\mathrm{E} \beta \mathrm{f}$ increased in the Oe rice lines compared to the OWt plants $(P<0.05)$ (Figures 4A and 5). The amounts of limonene in $1 \mu \mathrm{L}$ extracts from the Oe6, Oe7, and OWt plants was $1.87 \pm 0.42,1.90 \pm 0.53$, and $0.99 \pm 0.31 \mathrm{ng}$, respectively. Meanwhile, the amount of E $\beta \mathrm{f}$ in the Oe6, Oe7, and OWt plants was $10.62 \pm 4.04,12.70 \pm 3.09$, and $1.06 \pm 0.26 \mathrm{ng}$, respectively (Figure 4A). There was no significant difference in the amounts of limonene and $\mathrm{E} \beta \mathrm{f}$ between the $\mathrm{OWt}$ and $\mathrm{RWt}$ rice lines (Figure 4A).

Six other volatile compounds linalool, $(E)-\beta$-caryophyllene, methyl salicylate, $\alpha$-bisabolene, $(E)$ - $\alpha$-bergamotene, and $\alpha$-humulene were also increased significantly in the Oe rice lines compared to the OWt lines $(P<0.05)$. The content of these six volatiles in the OWt rice lines was extremely low (Figure 6). Also, these six volatile compounds were not detected in the $\mathrm{Ri}$ and RWt rice plants (Supplemental Figure S2). Among the six volatile compounds, the amount of linalool in $1 \mu \mathrm{L}$ of extract from the Oe6 and Oe7 rice lines was $4.97 \pm 2.58 \mathrm{ng}$, and $6.10 \pm 2.38 \mathrm{ng}$, respectively (Figure 4B). The amount of $(E)$ $\beta$-caryophyllene, $\alpha$-bisabolene, $(E)$ - $\alpha$-bergamotene, $\alpha$-humulene in the Oe6 rice lines was $1.54 \pm 0.27,1.80 \pm 0.19,1.61 \pm 0.29$, and $2.88 \pm 0.58 \mathrm{ng}$, separately. The amount of $(E)-\beta$-caryophyllene, $\alpha$-bisabolene, $(E)$ - $\alpha$-bergamotene, $\alpha$-humulene in the Oe7 lines was $1.53 \pm 0.37,2.05 \pm 1.24,1.24 \pm 0.44$, and $1.82 \pm 0.71 \mathrm{ng}$, respectively (Figure $4 \mathbf{B}$ ). Moreover, among the six volatile compounds, there were significant differences in the amount of methyl salicylate between the Oe6 and Oe7 lines $(P<0.05)$, and the amount of methyl salicylate in the Oe6 and Oe7 rice lines was $1.27 \pm 0.24$, and $3.65 \pm 1.04 \mathrm{ng}$, respectively (Figure 4B).

\section{DISCUSSION}

The current study shows that rice tps46 (Os08g0167800) is a key gene responsible for biosynthesis of limonene, methyl salicylate, E $\beta f,(E)-\beta$-caryophyllene, $\alpha$-bisabolene, $(E)$ $\alpha$-bergamotene, and $\alpha$-humulene from FPP. Among these volatiles, constitutive emissions of limonene and E $\beta f$ may due to the constitutive expressions of tps46 under natural conditions in rice. Furthermore, it is shown that silencing expression of tps46 makes rice vulnerable to attack by a herbivore, $R$. padi, which does not usually attack wild type rice. This finding suggests that TPS46 plays an important role in rice innate immunity to aphids.

Three TPS enzymes (Os02g02930, TPS46, and Os08g04500) are involved in the synthesis of the main terpene released from S. frugiperda-damaged rice plants (Yuan et al., 2008). All of the volatile components catalyzed by the above three recombinant enzymes in the E. coli-expression system only appeared in the blend of rice volatiles induced by $S$. frugiperda. The volatile product of recombinant Os02g02930 was (S)-linalool, and the volatile components of recombinant Os08g04500 were $\beta$-elemene, (E)- $\beta$-caryophyllene, $\alpha$-humulene, and germacrene (Yuan et al., 2008). However, the expression of tps46 was also found in untreated rice plants (Yuan et al., 2008; Sun et al.,
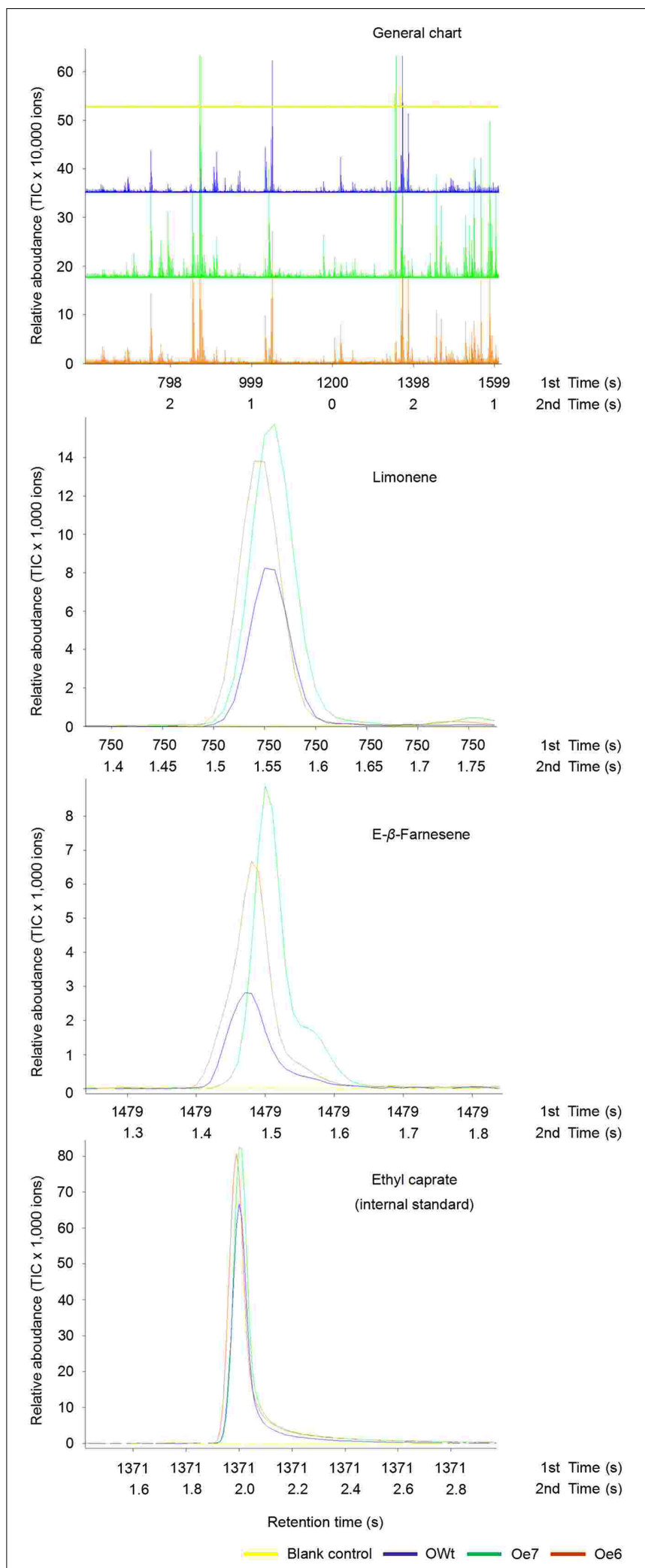

FIGURE 5 | Representative ToFMS of head-space volatile compounds from different rice lines at beginning of jointing-booting stage. Oe6 and 7 were the positive T1 tps46-Overexpression rice lines; OWt were the segregation lines of Oe transgenic plants. 


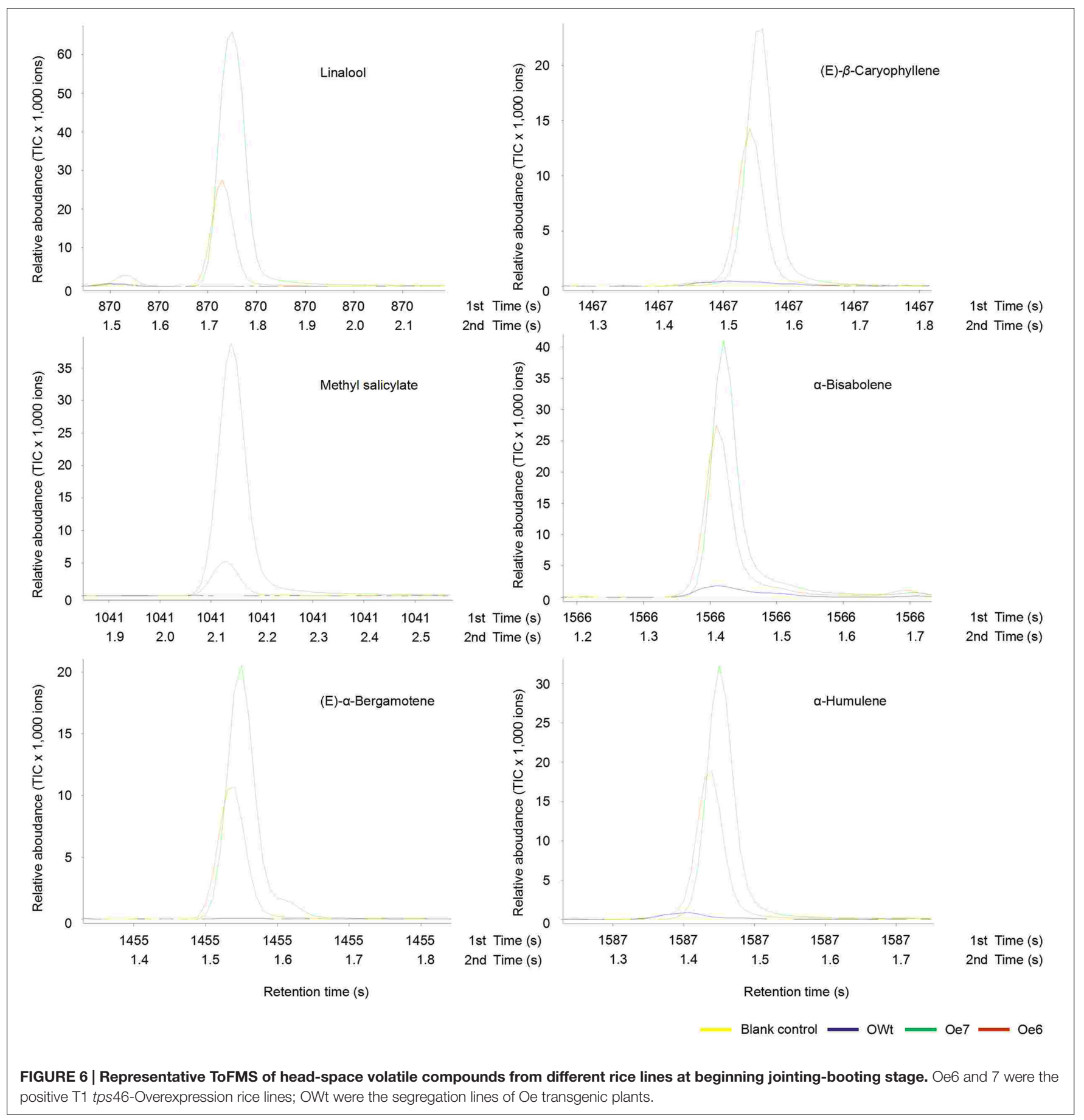

2011). In our Ri and Oe trials, eight volatile compounds were confirmed to be regulated by TPS46 in rice plants (Figures 5 and 6; Supplemental Figure S2), and these compounds are known to be involved in the indirect defense of Gramineous plants against phytophagous pests (Degenhardt et al., 2003; Lou et al., 2005, 2006; Rasmann et al., 2005; Schnee et al., 2006; Yuan et al., 2008). Among these eight volatiles, six compounds, linalool, (E)- $\beta$-caryophyllene, methyl salicylate, $\alpha$-bisabolene, $(E)$ - $\alpha$-bergamotene, and $\alpha$-humulene were only emitted in tps46-overexpression lines. These six volatiles were also only found in the blend of volatile compounds from rice plants infested by S. frugiperda (Yuan et al., 2008). Interestingly, (E)- $\beta$-caryophyllene and $\alpha$-humulene were the products of recombinant Os08g04500, whereas linalool was the main product of recombinant Os02g02930, and only $(E)$ - $\alpha$-bergamotene was the products of recombinant TPS46 (Yuan et al., 2008). In addition, suppression of emission of limonene and $\mathrm{E} \beta \mathrm{f}$ in $\mathrm{Ri}$ rice lines suggested that the biosynthesis of these two volatile 
compounds is regulated by tps46 in rice plants. Yuan et al. (2008) found that E $\beta f$ was the main product of recombinant TPS46 (Yuan et al., 2008). The results of this study revealed that the products of the same TPS in plants may be different from the TPS in the E. coli-expressed system. These differences could possibly be attributed to the complexity of terpene biosynthesis in rice. The constitutive emission of limonene and $\mathrm{E} \beta \mathrm{f}$ in untreated rice plants may be attributed to the expression of tps46 in the same plants.

It has been suggested that constitutive release of defensive volatiles should occur when plants are growing in an environment where there is a high probability of attack by herbivores (Degenhardt et al., 2003; Turlings and Ton, 2006). The constitutive release of volatiles is not only costly to plants, thereby resulting in yield declines but also inconsistent with the objectives of indirect defenses in plants because natural enemies do not get an "honest" signal indicating presence of prey (Degenhardt et al., 2003; Yamauchi et al., 2015). Nevertheless, there are several reports suggested that the release of volatiles is not necessarily very costly (Aharoni et al., 2005), and various plants constitute release some of the compounds of interests (Turlings and Ton, 2006). The nicest demonstration that constitutive emission might benefit pest control comes from the famous so-called "push-pull" studies by Khan et al. (1997), which also indicted that the constitutive emission volatiles from plants were also very important for plant defense against herbivore damage.

Although RWt and OWt were continuously cultivated in field or greenhouse for many years, few $R$. padi were found on these rice plants. In the present study, artificial infestation bioassays were consistent with the results of initial $R$. padi population survey in the greenhouse. After artificial infestation for 3 days, only approximately $20 \%$ of $R$. padi were observed on RWt and OWt rice lines, which indicated that they have some natural resistance to $R$. padi. Compared to control rice lines, $R$. padi significantly preferred to infest tps46-Ri rice lines (Figures 1-3). Differences in aphid infestation appear to be linked to differences in volatile production. For example, emission of two major volatiles, limonene and Eßf, was significantly reduced in the $\mathrm{Ri}$ lines (Figure 4A). These results indicate that limonene and $\mathrm{E} \beta \mathrm{f}$ may play a role in rice defenses against $R$. padi. However, to the best of our knowledge, there are almost no reports on roles of limonene in plant defenses against aphids. Our preliminary behavior assays also indicated that there was no obvious taxis response of $R$. padi to limonene stimuli at different doses (unpublished). Unlike limonene, E $\beta f$ was the main component of the alarm pheromone of $R$. padi (Nault and Bowers, 1974). In this work, the $R$. padi were significantly repelled by tps46-Oe rice lines $(P<0.05)$ (Figures 2 and 3 ), and the amount of E $\beta f$ was also significantly higher than the amount in control rice plants $(P<0.05)$ (Figure 4A). One hypothesis could be that E $\beta f$ has activity against aphids because it is the main component of the alarm pheromone for many aphids (Pickett et al., 2013). Eßf is secreted from the cornicle of aphids and causes other aphids in the vicinity to stop feeding, move away, and drop off the plant (Bernasconi et al., 1998; Hardie et al., 1999; de Vos and Jander, 2010).
Genetic engineering technique could regulate plants to emit E $\beta f$ to repel aphids (Beale et al., 2006; Gao et al., 2015). However, in the current study, we found that $\mathrm{E} \beta \mathrm{f}$ was released with other compounds that might inhibit alarm pheromone activity (Bruce et al., 2005a) and constitutive emission of E $\beta f$ from transgenic wheat did not reduce aphid populations in field experiments (Bruce et al., 2015). Another possibility is that the blend of compounds released from wild type rice expressing tps46 interferes with host recognition by the aphid (Bruce et al., 2005b).

In summary, the constitutive emission of E $\beta f$ and limonene from rice was regulated byTPS46. The tps46 was an important gene for rice defense against $R$. padi. Along with the development of insect-resistant transgenic plants (Wu et al., 2008), heterologous expression of TPS46 could be used as a potential strategy for improving the resistance of other crop species to aphids.

\section{AUTHOR CONTRIBUTIONS}

$\mathrm{YZ}, \mathrm{KW}$, and YG conceived and designed the experiments. $\mathrm{YS}, \mathrm{YN}$, and FQ performed transgenic rice experiments. YS, $\mathrm{QX}$, and $\mathrm{YZ}$ performed bioassay of $R$. padi performance experiments. YS, XH, WJ, and YZ performed volatile collection and identification experiments; $Y S$ and $Y Z$ analyzed the data and wrote the paper; TB revised the paper.

\section{ACKNOWLEDGMENT}

This work was supported by the China National "973" Basic Research Program (2012CB114104) and the National Natural Science Foundation of China $(31272048$, 31471778, 31672038, and 31621064).

\section{SUPPLEMENTARY MATERIAL}

The Supplementary Material for this article can be found online at: http://journal.frontiersin.org/article/10.3389/fpls.2017.00110/ full\#supplementary-material

FIGURE S1 | The relative expressions of tps46 in different rice lines at the late tilling stage. Oe1, 4, 6, 7, 8, 9 and 11 were the positive tps46-

Overexpression rice lines; OWt were the segregation lines of Oe transgenic plants, and 1, 2, 3 were the seeds obtained from three different maternal rice plants. Different lowercase letters above each bar indicate statistical difference with a statistical analysis system (SAS) followed by the Duncan's multiple comparison test $(p<0.05)$.

FIGURE S2 | Representative GC-MS of head-space volatile compounds from different rice lines at beginning of jointing-booting stage. (A) Positive T1 tps46-RNAi line rice plants. (B) The segregation lines of Ri transgenic plants. (C) The standard sample of limonene. (D) The standard sample of (E)- $\beta$-farnesene.

FIGURE S3 | The variation trends of Rhopalosiphum padi population numbers on T1 Ri line and RWt rice plants at 1-31 days of jointing-booting stage with another repeats. Different lowercase letters above each bar indicate statistical difference with a statistical analysis system (SAS) followed by the Duncan's multiple comparison test $(P<0.05)$. 


\section{REFERENCES}

Aharoni, A., Jongsma, M. A., and Bouwmeester, H. J. (2005). Volatile science? Metabolic engineering of terpenoids in plants. Trends Plant Sci. 10, 594-602. doi: 10.1016/j.tplants.2005.10.005

Balkema-Boomstra, A. G., Zijlstra, S., Verstappen, F. W., Inggamer, H., Mercke, P. E., Jongsma, M. A., et al. (2003). Role of cucurbitacin C in resistance to spider mite (Tetranychus urticae) in cucumber (Cucumis sativus L.). J. Chem. Ecol. 29, 225-235. doi: 10.1023/A:1021945101308

Beale, M. H., Birkett, M. A., Bruce, T. J. A., Chamberlain, K., Field, L. M., Huttly, A. K., et al. (2006). Aphid alarm pheromone produced by transgenic plants affects aphid and parasitoid behavior. Proc. Natl. Acad. Sci. U.S.A. 103, 10509-10513. doi: 10.1073/pnas.0603998103

Bernasconi, M. L., Turlings, T. C. J., Ambrosetti, L., Bassetti, P., and Dorn, S. (1998). Herbivore-induced emissions of maize volatiles repel the corn leaf aphid, shape Rhopalosiphum maidis. Entomol. Exp. Appl. 87, 133-142. doi: 10.1046/j.1570-7458.1998.00315.x

Bruce, T. J., Birkett, M. A., Blande, J., Hooper, A. M., Martin, J. L., Khambay, B., et al. (2005a). Response of economically important aphids to components of Hemizygia petiolata essential oil. Pest Manag. Sci. 61, 1115-1121. doi: 10.1002/ ps. 1102

Bruce, T. J., Wadhams, L. J., and Woodcock, C. M. (2005b). Insect host location: a volatile situation. Trends Plant Sci. 10, 269-274. doi: 10.1016/j.tplants.2005. 04.003

Bruce, T. J. A., Aradottir, G. I., Smart, L. E., Martin, J. L., Caulfield, J. C., Doherty, A., et al. (2015). The first crop plant genetically engineered to release an insect pheromone for defence. Sci. Rep. 5:11183. doi: 10.1038/srep 11183

Chen, S., Songkumarn, P., Liu, J., and Wang, G. L. (2009). A versatile zero background T-vector system for gene cloning and functional genomics. Plant Physiol. 150, 1111-1121. doi: 10.1104/pp.109.137125

Cheng, A. X., Xiang, C. Y., Li, J. X., Yang, C. Q., Hu, W. L., Wang, L. J., et al. (2007). The rice (E)-beta-caryophyllene synthase (OsTPS3) accounts for the major inducible volatile sesquiterpenes. Phytochemistry 68, 1632-1641. doi: 10.1016/j.phytochem.2007.04.008

de Vos, M., and Jander, G. (2010). Volatile communication in plant-aphid interactions. Curr. Opin. Plant Biol. 13, 366-371. doi: 10.1016/j.pbi.2010.05.001

Degenhardt, J. (2009). Indirect defense responses to herbivory in grasses. Plant Physiol. 149, 96-102. doi: 10.1104/pp.108.128975

Degenhardt, J., Gershenzon, J., Baldwin, I. T., and Kessler, A. (2003). Attracting friends to feast on foes: engineering terpene emission to make crop plants more attractive to herbivore enemies. Curr. Opin. Biotechnol. 14, 169-176. doi: 10.1016/S0958-1669(03)00025-9

Gao, L., Zhang, X. T., Zhou, F., Chen, H., and Lin, Y. J. (2015). Expression of a peppermint (E)- $\beta$-Farnesene synthase gene in rice has significant repelling effect on bird cherry-oat aphid (Rhopalosiphum padi). Plant Mol. Biol. Rep. 33, 1967-1974. doi: 10.1007/s11105-015-0888-4

Gaquerel, E., Weinhold, A., and Baldwin, I. T. (2009). Molecular interactions between the specialist herbivore Manduca sexta (Lepidoptera, Sphigidae) and its natural host Nicotiana attenuata. VIII. An unbiased GCxGC-ToFMS analysis of the plant's elicited volatile emissions. Plant Physiol. 149, 1408-1423. doi: 10.1104/pp.108.130799

Hardie, J., Pickett, J. A., Pow, E. M., and Smiley, D. W. M. (1999). “Aphids”, in Pheromones of Non-Lepidopteran Insects Associated with Agricultural Plants, eds J. Hardie and A. K. Minks (Wallingford: CAB International), 227-250.

Huang, X. Z., Chen, J. Y., Xiao, H. J., Xiao, Y. T., Wu, J., Wu, J. X., et al. (2015). Dynamic transcriptome analysis and volatile profiling of Gossypium hirsutum in response to the cotton bollworm Helicoverpa armigera. Sci. Rep. 5:11867. doi: $10.1038 /$ srep 11867

Jain, M., Nijhawan, A., Tyagi, A. K., and Khurana, J. P. (2006). Validation of housekeeping genes as internal control for studying gene expression in rice by quantitative real-time PCR. Biochem. Biophys. Res. Commun. 345, 646-651. doi: $10.1016 /$ j.bbrc.2006.04.140

Khan, Z. R., AmpongNyarko, K., Chiliswa, P., Hassanali, A., Kimani, S., Lwande, W., et al. (1997). Intercropping increases parasitism of pests. Nature 388, 631-632. doi: 10.1038/41681

Köllner, T. G., Held, M., Lenk, C., Hiltpold, I., Turlings, T. C. J., Gershenzon, J., et al. (2008). A maize (E)- $\beta$-caryophyllene synthase implicated in indirect defense responses against herbivores is not expressed inmost American maize varieties. Plant Cell 20, 482-494. doi: 10.1007/s11105-015-0888-4

Kong, Z. S., Li, M. N., Yang, W. Q., Xu, W. Y., and Xue, Y. B. (2006). A novel nuclear-localized CCCH-type zinc finger protein, OsDOS, is involved in delaying leaf senescence in rice. Plant Physiol. 141, 1376-1388. doi: 10.1104/ pp.106.082941

Livak, K. J., and Schmittgen, T. D. (2001). Analysis of relative gene expression data using real-time quantitative PCR and the 2- $\Delta \Delta \mathrm{CT}$ method. Methods 25, 402-408. doi: 10.1006/meth.2001.1262

Lou, Y. G., Hua, X. Y., Turlings, T. C. J., Cheng, J. A., Chen, X. X., and Ye, G. Y. (2006). Differences in induced volatile emissions among rice varieties result in differential attraction and parasitism of Nilaparvata lugens eggs by the parasitoid Anagrus nilaparvatae in the field. J. Chem. Ecol. 32, 2375-2387. doi: 10.1007/s10886-006-9151-7

Lou, Y. G., Ma, B., and Cheng, J. A. (2005). Attraction of the parasitoid Anagrus nilaparvatae to rice volatiles induced by the rice brown planthopper Nilaparvata lugens. J. Chem. Ecol. 31, 2357-2372. doi: 10.1007/s10886-0057106-z

Lu, Y. H., He, Y. P., and Gao, X. W. (2013). Comparative studies on acetylcholinesterase characteristics between the aphids, Sitobion avenae and Rhopalosiphum padi. J. Insect Sci. 13, 1-9. doi: 10.1673/031.013.0901

Nagegowda, D. A., Gutensohn, M., Wilkerson, C. G., and Dudareva, N. (2008). Two nearly identical terpene synthases catalyze the formation of nerolidol and linalool in snapdragon flowers. Plant J. 55, 224-239. doi: 10.1111/j.1365-313X. 2008.03496.x

Nault, L. R., and Bowers, W. S. (1974). Multiple alarm pheromones in aphids. Entomol. Exp. Appl. 17, 455-457. doi: 10.1111/j.1570-7458.1974.tb00369.x

Park, C. H., Chen, S. B., Shirsekar, G., Zhou, B., Khang, C. H., Songkumarn, P., et al. (2012). The Magnaporthe oryzae effector AvrPiz-t targets the RING E3 ubiquitin ligase APIP6 to suppress pathogen-associated molecular patterntriggered immunity in rice. Plant Cell 24, 4748-4762. doi: 10.1105/tpc.112. 105429

Pichersky, E., and Gershenzon, J. (2002). The formation and function of plant volatiles: perfumes for pollinator attraction and defense. Curr. Opin. Plant Biol. 5, 237-243. doi: 10.1016/S1369-5266(02)00251-0

Pickett, J. A., Allemann, R. K., and Birkett, M. A. (2013). The semiochemistry of aphids. Nat. Prod. Rep. 30, 1277-1283. doi: 10.1039/c3np70036d

Qu, S., Liu, G., Zhou, B., Bellizzi, M., Zeng, L., Dai, L., et al. (2006). The broadspectrum blast resistance gene $\mathrm{Pi} 9$ encodes a nucleotide-binding site-leucinerich repeat protein and is a member of a multigene family in rice. Genetics 172 , 1901-1914. doi: 10.1534/genetics.105.044891

Rasmann, S., Köllner, T. G., Degenhardt, J., Hiltpol, I., Toepfer, S., Kuhlmann, U., et al. (2005). Recruitment of entomopathogenic nematodes by insect-damaged maize roots. Nature 434, 732-737. doi: 10.1038/nature03451

Schnee, C., Köllner, T. G., Gershenzon, J., and Degenhardt, J. (2002). The maize gene terpene synthase 1 encodes a sesquiterpene synthase catalyzing the formation of (E)-beta-farnesene, (E)-nerolidol, and (E,E)-farnesol after herbivore damage. Plant Physiol. 130, 2049-2060. doi: 10.1104/pp.008326

Schnee, C., Köllner, T. G., Held, M., Turlings, T. C., Gershenzon, J., and Degenhardt, J. (2006). The products of a single maize sesquiterpene synthase form a volatile defense signal that attracts natural enemies of maize herbivores. Proc. Natl. Acad. Sci. U.S.A. 103, 1129-1134. doi: 10.1073/pnas.0508027103

Sun, Y., Sheng, Y., Bai, L. X., Zhang, Y. J., Xiao, Y. F., Xiao, L. B., et al. (2014). Characterizing heat shock protein 90 gene of Apolygus lucorum (Meyer-Dür) and its expression in response to different temperature and pesticide stresses. Cell Stress Chaperones 19, 725-739. doi: 10.1007/s12192-014-0500-0

Sun, Y., Zhang, Y. J., Cao, G. C., Wu, K. M., Gao, X. W., and Guo, Y. Y. (2011). Rice gene expression profiles responding to larval feeding of the striped stem borer at the 1st to 2nd instar stage. Insect Sci. 18, 273-281. doi: 10.1111/j.1744-7917. 2010.01372.x

Turlings, T. C. J., and Ton, J. (2006). Exploiting scents of distress: the prospect of manipulating herbivore-induced plant odors to enhance the control of agricultural pests. Curr. Opin. Plant Biol. 9, 421-427. doi: 10.1016/j.pbi.2006. 05.010

Turlings, T. C. J., Tumlinson, J. H., Heath, R. R., Proveaux, A. T., and Doolittle, R. E. (1991). Isolation and identification of allelochemicals that attract the larval parasitoid, Cotesia marginiventris (Cresson), to the microhabitat of one of its hosts. J. Chem. Ecol. 17, 2235-2251. doi: 10.1007/BF00988004 
Wang, Z., Chen, C. B., Xu, Y. Y., Jiang, R. X., Han, Y. E., Xu, Z. H., et al. (2004). A practical vector for efficient knockdown of gene expression in rice (Oryza sativa L.). Plant Mol. Biol. Rep. 22, 409-417. doi: 10.1007/BF027 72683

Wu, K. M., Lu, Y. H., Feng, H. Q., Jiang, Y. Y., and Zhao, J. Z. (2008), Suppression of cotton bollworm in multiple crops in China in areas with Bt toxin-containing cotton. Science 321, 1676-1678. doi: 10.1126/science. 1160550

Yamauchi, A., van-Baalen, M., Kobayashi, Y., Takabayashi, J., Shiojiri, K. W., and Sabelis, M. (2015). Cry-wolf signals emerging from co-evolutionary feedbacks in a tritrophic system. Proc. Biol. Sci. 282:20152169. doi: 10.1098/rspb.2015. 2169

Yu, H. L., Zhang, Y. J., Wyckhuys, K. A. G., Wu, K. M., Gao, X. W., and Guo, Y. Y. (2010). Electrophysiological and behavioral responses of Microplitis mediator (Hymenoptera: Braconidae) to caterpillar induced volatiles from cotton. Environ. Entomol. 39, 600-609. doi: 10.1603/EN09162
Yuan, J. S., Köllner, T. G., Wiggins, G., Grant, J., Degenhardt, J., and Chen, F. (2008). Molecular and genomic basis of volatile-mediated indirect defense against insects in rice. Plant J. 55, 491-503. doi: 10.1111/j.1365-313X.2008. 03524.x

Conflict of Interest Statement: The authors declare that the research was conducted in the absence of any commercial or financial relationships that could be construed as a potential conflict of interest.

Copyright $\odot 2017$ Sun, Huang, Ning, Jing, Bruce, Qi, Xu, Wu, Zhang and Guo. This is an open-access article distributed under the terms of the Creative Commons Attribution License (CC BY). The use, distribution or reproduction in other forums is permitted, provided the original author(s) or licensor are credited and that the original publication in this journal is cited, in accordance with accepted academic practice. No use, distribution or reproduction is permitted which does not comply with these terms. 